\title{
Inverter Control Analysis in a Microgrid Community Based on Droop Control Strategy
}

\author{
Navid Salehi, Herminio Martínez-García, Guillermo Velasco-Quesada and Encarna García-Vílchez \\ Department of Electronic Engineering \\ Escola d'Enginyeria de Barcelona Est (EEBE), Technical University of Catalonia (UPC)-BarcelonaTech \\ Campus of Diagonal-Besòs, 08019 Barcelona (Spain) \\ Phone/Fax number:+0034.93.413.72.90, e-mail: navid.salehi@upc.edu, herminio.martinez@upc.edu
}

\begin{abstract}
Power-sharing in a microgrid community (MGC) requires mandatory strategies in order to stabilize the system by maintaining the rated frequency and voltage. The hierarchical control strategy is the most adopted control structure due to providing seamless operation in transient between islanded and grid-connected modes. Droop control strategy is discussed in this paper to control the voltage source inverter (VSI) in power exchange mode with other microgrids (MGs) or main utility grid. After analysing the average model of three-phase VSI, the voltage and current transfer functions are obtained according to the considered droop controller. The stability and seamless operation of the system are analysed, and the simulation results verify the control loops of the VSI can handle the power variation of the MG effectively.
\end{abstract}

Key words. Microgrid Community (MGC), Voltage Source Inverter (VSI), Inner loop, Outer loop, Droop control.

\section{Introduction}

The control loops of inverters in the microgrids community (MGC) are more complicated from the stability point of view since, in addition to the voltage amplitude and frequency variations, the exchange power among microgrids (MGs) or main grid can affect the stability of the inverter [1], [2]. For this reason, multiple control strategies are proposed to control the MGC in recent years. Master-slave, peer-to-peer, and hierarchical control strategy are the most well-known structures in MGC [3]. In this paper, hierarchical control is applied to control the MGC in order to take the advantage of stable operation of the system especially at the transition between islanded and grid-connected mode [4].

The hierarchical control structure consists of three control levels: primary, secondary, and tertiary control [3]. In addition to inverter control loops, the droop controller in the primary control level is responsible to provide voltage and frequency stability [5]. Different droop control methods with potential pros and cons are surveyed in [6]. Moreover, further researches are performed to improve the transient response of the droop controller. Utilizing virtual inductance and real-time integration filter is proposed in [7]. In [8], inaccurate reactive power sharing due to the effect of feeder impedance is addressed as a drawback of the droop control method. To tackle this matter, conventional droop characteristics and a proportional-resonant (PR) controller are used. Moreover, the problem caused by imprecise frequency measurement is suppressed by proposing an improved phase-locked loop (PLL).

Moreover, an energy management system (EMS) at the secondary control level can provide an optimal and efficient operation of the whole system according to the interlink communication infrastructure. The centralized, decentralized, hybrid, and distributed are various control architecture which used in data commination in MGC [9]. The advantages and disadvantages of these communication topologies are reviewed in [10]. Finally, the optimal power flow, optimal economic operation, and reactive power supervision are under control at the tertiary level.

In this paper, firstly, the droop control method is introduced to identify the effect of power-sharing among MGs or the main grid. Then, the inverter control structure with inner loop and outer loop is demonstrated. The active and reactive power references of the control loops are able to be adjusted according to the droop control methods (P$\omega, \mathrm{Q}-\mathrm{V})$, which affects the inverter control loops. In this paper, the incorporation of the droop controller into the inverter control loops is analysed and the voltage and current transfer functions are obtained considering the droop characteristics. Consequently, to achieve the PI compensator parameters the average model of a threephase voltage source inverter (VSI) is presented. Finally, the simulation results of a VSI are presented and the results verify that the control loops of the inverter can stabilize the system effectively.

\section{Steady-State Operation Based on Droop Control}

Fig. 1 shows an MGC consisting of three MGs that can operate in grid-connected or islanding operation mode. According to the hierarchical coordination strategy of the MGC, each MG can exchange power with the main grid 
or other MGs in order to achieve the effective and efficient operation of the whole system. Therefore, maintaining the rated frequency and voltage, and power balance equilibrium of each $\mathrm{MG}$ can be optimally analysed by an EMS to guarantee stability and optimum system operation.

Inverters play a crucial role specifically in $\mathrm{MG}$ control strategies [6]. Fig. 2 illustrates the energy generation units in $\mathrm{MG \# 1}$ consisting of photovoltaic (PV), wind turbine (WT), and energy storage system (ESS). The bus $B_{2}$ in Fig. 2 can be considered as a point of common coupling (PCC) shown in Fig. 1. The bus $B_{2}$ is connected to the bus $B_{1}$ of MG\#1 by feeder line impedance Z. In (1) the power flow from $B_{1}$ to $B_{2}$ is shown (for simplicity the resistance is neglected):

$$
P_{12}=\frac{\left|V_{1}\right|\left|V_{2}\right|}{X} \sin \left(\delta_{1}-\delta_{2}\right)
$$

where $P_{12}$ is the active power flow from bus $B_{1}$ to $B_{2}$ and $X=2 \pi f L$ is the reactance of the line. As it can be inferred from (1), in order to transfer the power from $B_{1}$ to $B_{2}, \delta_{1}$ has to be greater than $\delta_{2}$. In addition, $V_{1}$ and $\delta_{1}$ are the voltage and the phase angle of the inverters, respectively. Therefore, in order to control the active power $P_{12}$ both amplitude voltage $\left(V_{l}\right)$ and phase angle $\left(\delta_{l}\right)$ can be controlled.

Fig. 3 depicts the variation of the phase angle $\delta$ (and $V$ ) by changing the active power $P$ (and $Q$ ). The droop characteristics of an MG are represented in (2) and (3):

$$
\begin{gathered}
f^{\text {rated }}-f=m_{P}\left(P-P^{\text {rated }}\right) \\
V^{\text {rated }}-V=m_{Q}\left(Q-Q^{\text {rated }}\right)
\end{gathered}
$$

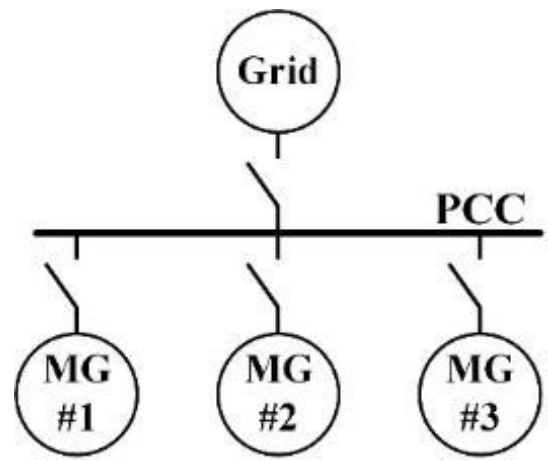

Fig. 1. Microgrid community

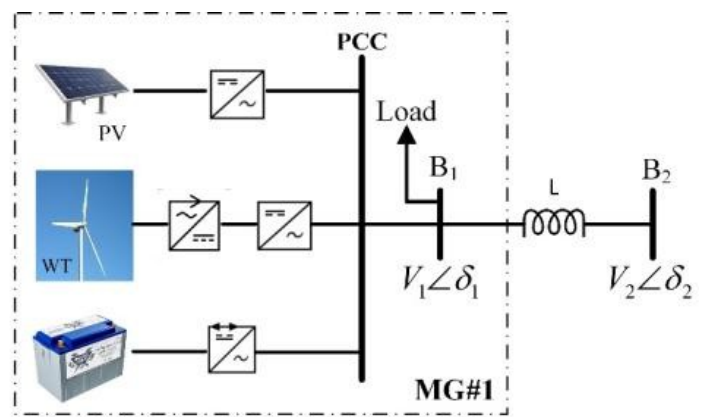

Fig. 2. A sample microgrid (MG\#1) consists of PV, WT and battery

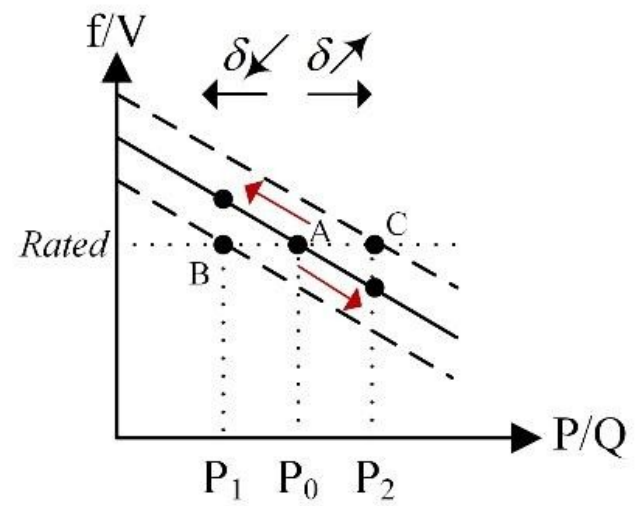

Fig. 3: Droop control strategy

where $m_{P}$ and $m_{Q}$ are the droop coefficient that determines the control sensitivity. According to (1) and (2), by controlling the active power $P$ and reactive power $Q$, the frequency and voltage of the $\mathrm{MG}$ can be controlled, respectively. For instance, suppose that MG\#2 in Fig.1 needs more active power in order to supply the load and maintaining the rated frequency and voltage amplitude of the $\mathrm{MG}$, therefore, in order to compensate for the required power, supposed that MG\#1 in Fig. 2 is able to transfer

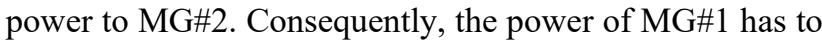
increase from $P_{0}$ (point A) to $P_{2}$ (point $\mathrm{C}$ ) and $\left(P_{2}-P_{0}\right)$ power transfer to $\mathrm{MG \# 2}$ to keep the frequency at the rated value. The same analysis can be performed for reactive power in order to keep the rated voltage of $\mathrm{MG}$

\section{Inverter Control}

To analyse the performance of the inverter in transition and steady-state operation, the average model of a threephase voltage source inverter is considered in this paper [10], [11]. Fig. 4 shows the control structure of the inverter. The analysis is performed in dq reference frame. The dq average model of three-phase VSI is presented in (4) and (5):

$$
\begin{aligned}
& L \frac{d i_{d}}{d t}=L \omega i_{q}+\left(d-\frac{1}{2}\right) V_{D C}-r . i_{d}-e_{d} \\
& L \frac{d i_{q}}{d t}=-L \omega i_{d}+\left(d-\frac{1}{2}\right) V_{D C}-r \cdot i_{q}-e_{q}
\end{aligned}
$$

where $L, r$, and $V_{D C}$ are determined in Fig. 4, $\omega=2 \pi f, d$ is PWM modulator gain $\left(d=v_{\text {mod }} / V_{P}\right.$ where $v_{\text {mod }}$ is modulation voltage, and $V_{P}$ is the peak value of carrier signal), $i_{d}$ and $i_{q}$ are the $d$ and $q$ input current component, and $e_{d}$ and $e_{q}$ are the $d$ and $q$ component of PCC. Fig. 5 illustrates the dq average model of the inverter.

As can be seen from Fig. 4, the inner current loop and outer voltage loop are applied to the control structure to control the output voltage of the inverter. The phase angle of the secondary side of the inverter calculated by the synchronous reference frame (SRF) PLL, which is used in order to transform the output voltage and current of the inverter from $a b c$ frame to $d q$. The SRF-PLL avoids the double frequency error problem of single-phase standard 


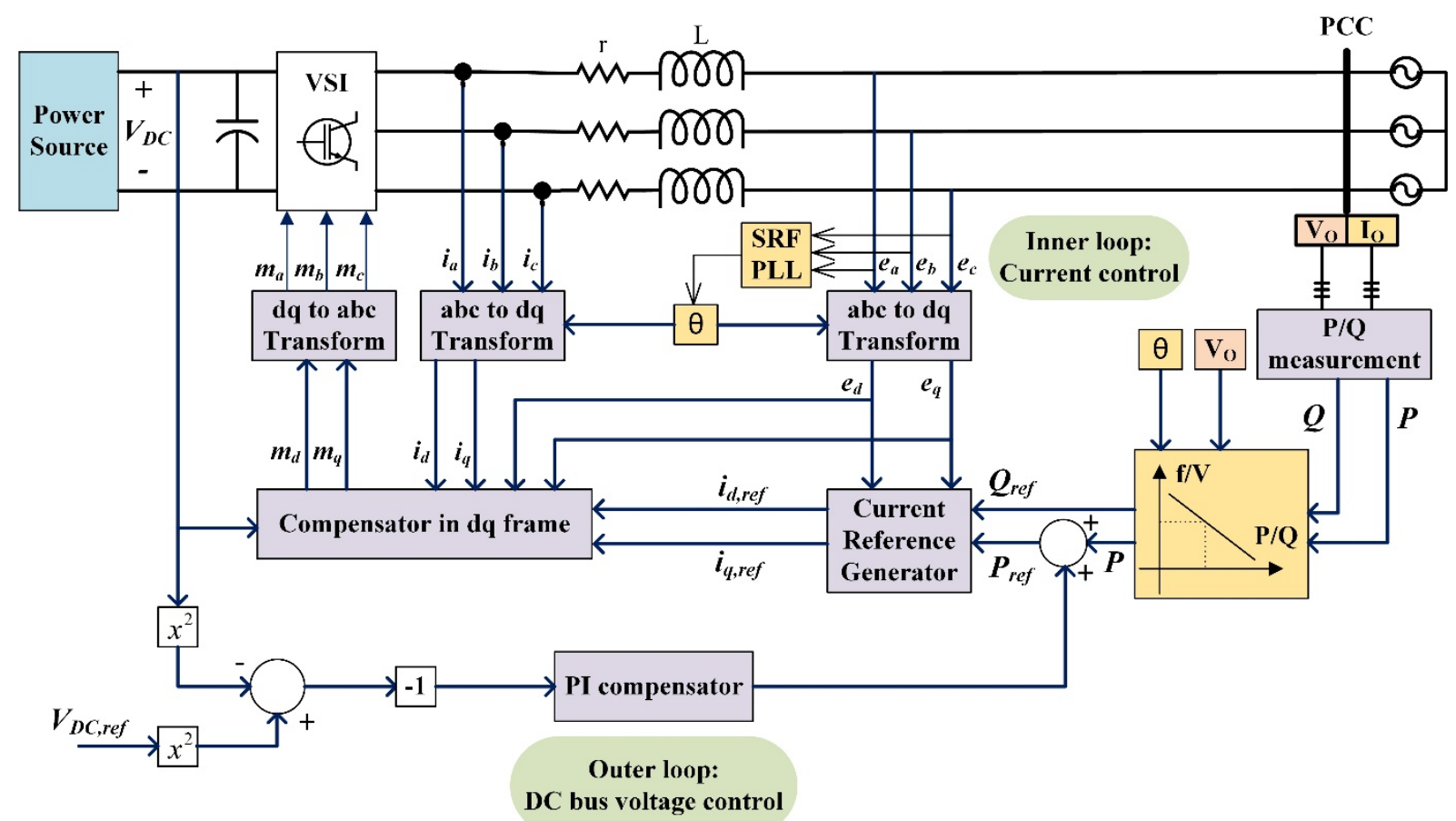

Fig. 4: Inner current loop and outer voltage loop applied to the control structure to control the output voltage of the inverter

PLL [12]. Fig. 6 shows the conventional SRF-PLL structure.

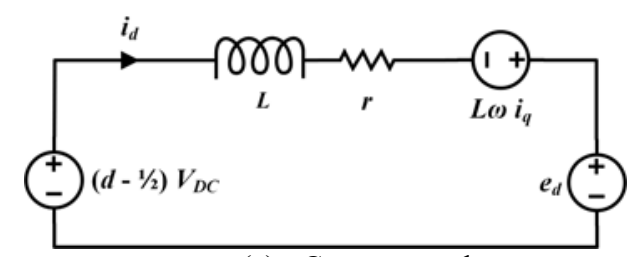

(a) Component $\mathrm{d}$

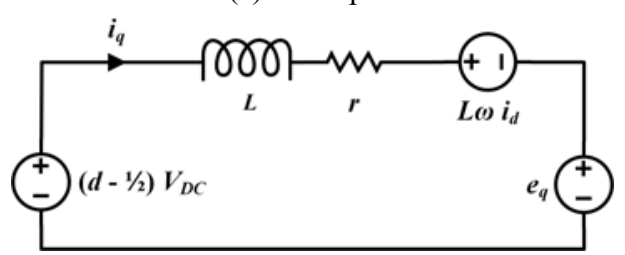

(a) Component $q$

Fig. 5: dq average model of three-phase VSI

In (6) and (7), the current reference generator in dq frame are presented:

$$
\begin{aligned}
i_{d, r e f} & =2 \cdot \frac{p e_{d}+q e_{q}}{e_{d}^{2}+e_{q}^{2}} \\
i_{q, r e f} & =2 \cdot \frac{p e_{q}-q e_{d}}{e_{d}^{2}+e_{q}^{2}}
\end{aligned}
$$

where, because SRF-PLL is aligned with the $\mathrm{d}$ axis, then $e_{q}=0$, and equations (6) and (7) can be simplified. Input DC voltage $\left(V_{D C}\right)$ or the square of the DC voltage $\left(V_{D C}{ }^{2}\right)$ is the state variable of the DC port which represents the energy stored in the DC capacitor. DC voltage loop transfer function is depicted in Fig. 7.

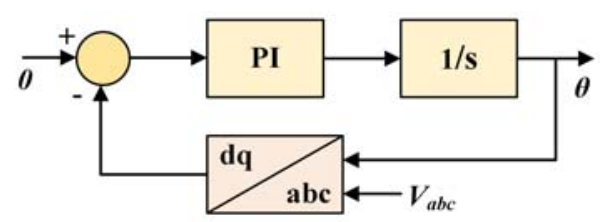

Fig. 6: Conventional SRF-PLL

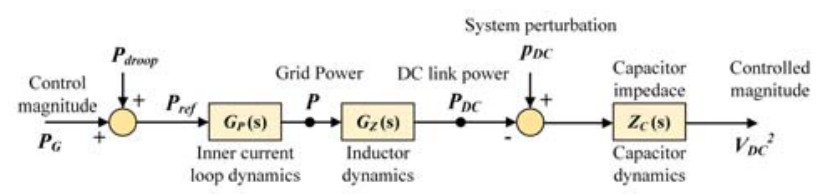

Fig. 7: Voltage control loop

Therefore, the transfer function of the voltage loop compensator can be calculated as:

$$
G_{V}(s)=\frac{V_{D C}^{2}}{P_{G}+P_{\text {droop }}}=G_{P}(s) \cdot G_{Z}(s) \cdot Z_{C}(s),
$$

where:

$$
\begin{gathered}
G_{P}=\frac{P}{P_{\text {ref }}+P_{\text {droop }}}=\frac{i}{i_{\text {ref }}}, \\
G_{Z}=\frac{P_{D C}}{P}=\left(1+\frac{s}{\omega_{z}}\right), \\
\omega_{Z}=\frac{3 E^{2}}{2 L P_{O}}, \\
Z_{C}=\frac{1}{(C / 2) s},
\end{gathered}
$$

and $E$ is the phase to neutral grid voltage amplitude and $P_{O}$ is the static value of the grid power. 
Furthermore, the current control loop in $\mathrm{d}-\mathrm{q}$ reference frame is depicted in Fig. 8.

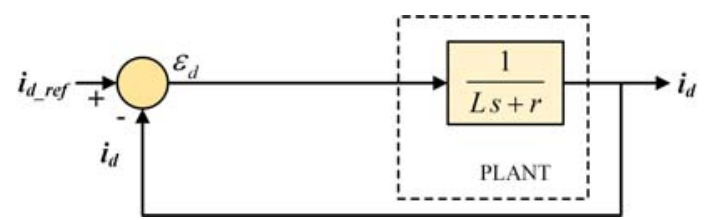

Fig. 8: Current control loop in d-q reference frame

$$
G_{i}(s)=\frac{1}{L s+r}
$$

In Table I, the characteristics of the inverter are defined. Eventually, according to the defined average model of three-phase VSI, and calculated voltage and current transfer functions, the PI compensators of the inverter are designed. To this purpose, firstly, the stability over the whole range of operation point to obtain the phase margin (PM), and, secondly, rejection of the perturbation in order to obtain the crossover frequency (fc) are considered. The results of calculations are presented in Table I.

Table I: Characteristics of the inverter.

\begin{tabular}{||c|c||c|c||}
\hline \hline Parameter & Value & Parameter & Value \\
\hline \hline$V_{p h-n}$ & $230 V_{r m s}$ & $L$ & $300 \mu \mathrm{H}$ \\
\hline$P_{O}$ & $4000 \mathrm{~W}$ & $C$ & $470 \mu \mathrm{F}$ \\
\hline \hline Current loop & Value & Voltage loop & Value \\
\hline$K_{i}$ & 2.34 & $K_{v}$ & 0.5 \\
\hline$T_{i}$ & $126.5 \mu \mathrm{s}$ & $T_{v}$ & $5 \mathrm{~ms}$ \\
\hline
\end{tabular}

\section{Simulation Results}

In order to analyse the operation of the inverter in power exchange mode between two MGs or the MG and the main utility grid, the average model of a VSI is used in Matlab/Simulink. As it can be seen from Fig. 4, in addition to the active and reactive power reference, the input voltage also can be varied. The main duty of voltage and current loops is stabilizing the inverter operation regarding the variation of reference values and input voltage $V_{D C}$. In this simulation, input DC voltage at $0.2 \mathrm{~s}$ changes from $500 \mathrm{~V}$ to $700 \mathrm{~V}$, and the active power reference at $0.3 \mathrm{~s}$ changes from $4 \mathrm{~kW}$ to $5.5 \mathrm{~W}$, however, the reactive power reference is maintained constant during the simulation time. Moreover, the simulation is done for two different situations in control loops by considering both $V_{D C}$ (input DC voltage) Feed-Forward (FF) and without $V_{D C} \mathrm{FF}$ in order to analyse the effect of $V_{D C} \mathrm{FF}$. Fig 9 shows the current loops with and without $V_{D C} \mathrm{FF}$ (in without $V_{D C} \mathrm{FF}$ mode, the $V_{D C} \mathrm{FF}$ is omitted). FeedForward circuit can provide the following advantages in the design and control of inverter:

- Complete linear system is obtained,

- Plant is only dependent on the inductor value,

- Design the compensator is easier,

- Optimum design for the compensator can be obtained.

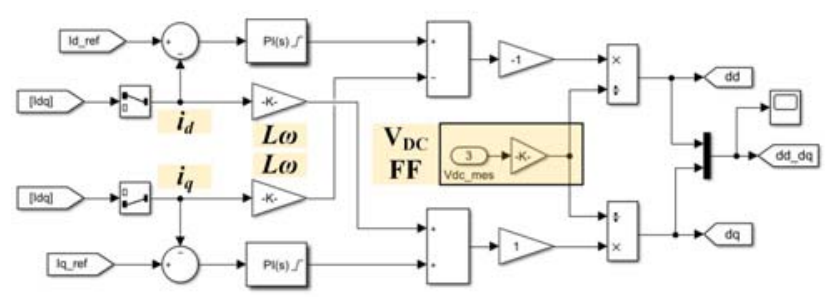

Fig. 9: Current loop with and without $V_{D C}$ Feed-Forward

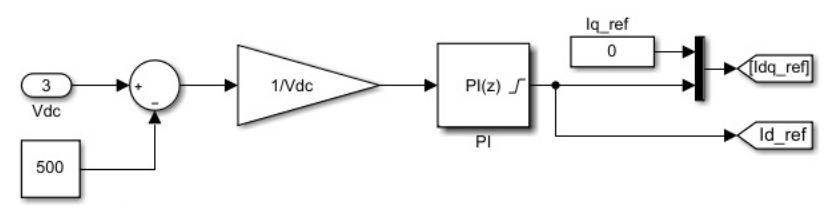

Fig. 10: Voltage loop

The reference value for $i_{d}$ and $i_{q}$ in Fig. 9 is calculated by the voltage loop and are compared with the measured values. Fig. 10 demonstrates the voltage loop in the Simulink. The values of PI compensators for voltage and current loops are stated in Table I, and Fig. 11 (from (a) to (1)) shows the current, and power of the inverter. Moreover, the $V_{D C}$ Feed-Forward effect can be seen in these figures. Figure 11-(a), (b) represent the inverter output current with and without FF, respectively. Fig. 11(g) shows the inverter power without FF, and Fig 11-(j) shows the inverter power without FF. Figure 11-(b), (e), $(\mathrm{h})$, and (k) are the magnified part of the curves related to the input voltage $V_{D C}$ reference change. Figure 11-(c), (f), (i), and (1) are related to the active power reference change. The simulation results show the $V_{D C}$ FeedForward can control the power and current more effectively by variation input DC voltage. In addition, the results verify the stabilization and seamless operation of the inverter by power reference changing at $0.3 \mathrm{~s}$ in order to transfer the extra power to another MG or main grid. The results show the inverter will be able to keep the stability after a short transition period of time especially in Feed-forward operation mode.

\section{Conclusion and future Work}

This paper investigates the droop control strategy in order to control the MG to maintain the frequency and voltage of the MG. Then, by considering the average model of a VSI, the inner and outer control loops with and without input voltage $V_{D C}$ Feed-Forward circuit are discussed. The control loops verify the stabilization of the inverter operation in case of changing the input voltage $V_{D C}$ reference, and active power reference of the control loops by droop controller. Eventually, the simulation in Matlab/Simulink shows that the designed voltage and current PI compensator make the VSI capable to handle the stability of the inverter based on the droop control strategy. The effect of $V_{D C} \mathrm{FF}$ is also presented in the simulation results, and the smooth operation of the inverter in the case of using $V_{D C} \mathrm{FF}$ can be observed. In future work, the theoretical considerations on system stability will be discussed. 

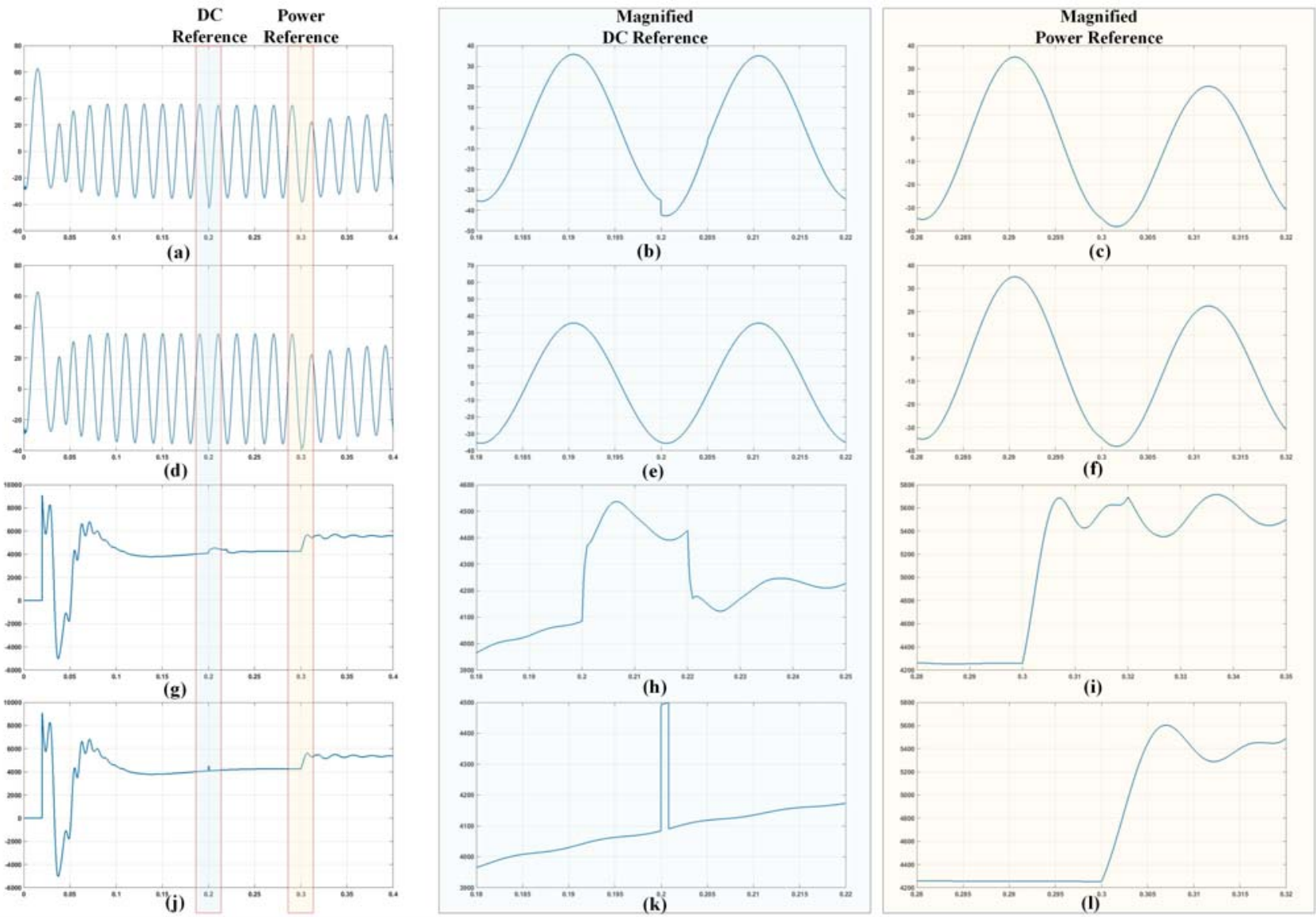

Fig. 11. Simulation results : (a), (d) inverter current with and without FF; (g), (h) inverter power with and without FF, (b), (e), (h), (k) magnified DC reference change, (c), (f), (i), (l) magnified power reference change

Moreover, the short transient time resulted from power reference variation verify the seamless and stable operation of the inverter in hierarchical control structure by adopting the droop control strategy.

\section{Acknowledgement}

This work has been partially supported by the Spanish Ministerio de Ciencia, Innovación y Universidades (MICINN)-Agencia Estatal de Investigación (AEI) and the European Regional Development Funds (FEDER), by project PGC2018-098946-B-I00.

\section{References}

[1] M.H. Andishgar, E. Gholipour, and R.A. Hooshmand, "An Overview of Control Approaches of Inverter-based Microgrids in Islanding Mode of Operation”, Renewable and Sustainable Energy Reviews, 80, 2017, paper 1043-1060.

[2] S. Xu, J. Wang, and J. Xu, "A Current Decoupling Parallel Control Strategy of Single-Phase Inverter with Voltage and Current Dual Closed-Loop Feedback", IEEE Transactions on Industrial Electronics, 60(4), 2011, paper1306-1313.

[3] Islam, Mojaharul, Fuwen Yang, and Mohammad Amin. "Control and optimisation of networked microgrids: A review." IET Renewable Power Generation 15, no. 6 (2021): 1133-1148.

[4] Abhishek, Anand, Aashish Ranjan, Sachin Devassy, Brijendra Kumar Verma, Subhash Kumar Ram, and Ajeet Kr Dhakar. "Review of hierarchical control strategies for DC microgrid." IET Renewable Power Generation 14, no. 10 (2020): 1631-1640.
[5] Bidram, Ali, and Ali Davoudi. "Hierarchical structure of microgrids control system." IEEE Transactions on Smart Grid 3, no. 4 (2012): 1963-1976.

[6] Alam, Mahamad Nabab, Saikat Chakrabarti, and Arindam Ghosh. "Networked microgrids: State-of-the-art and future perspectives." IEEE Transactions on Industrial Informatics 15, no. 3 (2018): 1238-1250.

[7] M.A. Abusara, S.M. Sharkh, and J.M. Guerrero, "Improved Droop Control Strategy for Grid-Connected Inverters", Sustainable Energy, Grids and Networks, 1, 2015, paper 1019.

[8] Zare, Ahmad, Mazda Moattari, and Rui Melicio. "Distributed Generation Control Using Modified PLL Based on Proportional-Resonant Controller." Applied Sciences 10, no. 24 (2020): 8891.

[9] Zhou, Quan, Mohammad Shahidehpour, Aleksi Paaso, Shay Bahramirad, Ahmed Alabdulwahab, and Abdullah Abusorrah. "Distributed control and communication strategies in networked microgrids." IEEE Communications Surveys \& Tutorials 22, no. 4 (2020): 2586-2633.

[10] Xing, Xiaowen, Lili Xie, and Hongmin Meng. "Cooperative energy management optimization based on distributed MPC in grid-connected microgrids community." International Journal of Electrical Power \& Energy Systems 107 (2019): 186-199.

[11] Ahmed, Sara, Zhiyu Shen, Paolo Mattavelli, Dushan Boroyevich, and Kamiar J. Karimi. "Small-signal model of voltage source inverter (VSI) and voltage source converter (VSC) considering the deadtime effect and space vector modulation types." IEEE Transactions on Power Electronics 32, no. 6 (2016): 4145-4156.

[12] Karimi-Ghartema, Masoud. Enhanced phase-locked loop structures for power and energy applications. John Wiley \& Sons, 2014. 